\title{
Sonodynamic and Photodynamic Therapy in Breast Cancer - A Pilot Study
}

\begin{abstract}
In this exploratory pilot study, we investigated the potential efficacy of a new systemic tumor therapeutic method as a supplementary or salvage treatment in advanced refractory breast cancer. Termed Sono-Photo Dynamic Therapy (SPDT), this technology utilizes full body and targeted ultrasound in conjunction with photodynamic therapy. Twelve patients with advanced breast carcinoma participated, eleven with metastases in the viscera and brain, and nine within bone. Nine patients had concurrent low dose chemotherapy with the SPDT. After the first round of treatment (where the number of cycles of SPDT varied from 1-4), the overall response rate was 75\% (two patients achieved Complete Response [16.67\%] and seven patients achieved Partial Response [58.33\%]). Results suggest that SPDT was not associated with serious side effects, and may enhance chemotherapy efficacy in the treatment of advanced refractory breast cancer. Despite the exploratory nature of this study, the results suggest that SPDT shows promise as a new systemic approach for tumor adjunct therapy and merits further investigation.
\end{abstract}

Keywords: Photodynamic therapy; Breast cancer; Chemotherapy; Federal drug administration; Reactive oxygen species; In-vivo; In vitro
Research Article

Volume 9 Issue 5 - 2017

Zhang $\mathrm{W}^{1 *}$, Li K ${ }^{1}$, Lu J ${ }^{1}$, Peng $\mathrm{Z}^{1}$, Wang $\mathrm{X}^{2 \#}$, Li $Q^{3}$, Zhao $G^{3}$, Hao $J^{3}$, Luo $Y^{4}$, Zhao $Y^{5}$, Yin $X^{6}$ and $\mathbf{O}^{\prime}$ Brien $\mathrm{KA}^{6-8}$

${ }^{1}$ Guangzhou Medical University of Chinese Medicine, China

${ }^{2}$ Department of Oncology, Guangzhou Liuhuaqiao Hospital, China

${ }^{3}$ EC Bio-tech Co Ltd, China

${ }^{4}$ South China Normal University, China

${ }^{5}$ Renkang Hospital, China

${ }^{6}$ Victoria University, Australia

${ }^{7}$ National Institute of Integrative Medicine, Australia

${ }^{8}$ National Institute of Complementary Medicine, Western

Sydney University, Australia

*Corresponding author: Wenyin Zhang, Department of Oncology, Jinshazhou Hospital, No 1, Lichuan Dong Jie, Jinshanzhou, Guanghzou, China,

Email: wyzhang08@163.com

"Xiaohuai Wang, Department of Oncology, Guangzhou

Liuhuaqiao Hospital, Guangzhou, Guangdong 510010, P. R.

China, Email: wangxhgz@163.com

Received: October 29, 2017 | Published: December 04, 2017 Disease; CR: Complete Response; PR: Partial Response; RECIST: Response Evaluation Criteria In Solid Tumors; OS: Overall Survival

\section{Introduction}

Photodynamic therapy (PDT) is an established therapeutic method that was first approved by the US Federal Drug Administration (FDA) for certain kinds of cancer in 1998. However, the successful application of this method has been limited to superficial lesions because of the limited penetration of laser light into tumor tissues [1,2]. Sonodynamic therapy (SDT) developed as a complementary or alternative therapy to PDT [3]. It uses a combination of low-intensity ultrasound and specialised chemical agents termed sonosensitizers, which are drugs that can be activated by ultrasound. Ultrasound is able to penetrate deeply into tissues and can be focused on a small region of tumor where it activates the sonosensitizer, creating a cascade of endogenous cytotoxic agents [4]. The advantage of ultrasound is that it penetrates more deeply than light, and therefore it is likely to be more effective for the treatment of deeper tumours than PDT [4].

So far, many photosensitizers have been found to have sonodynamic activity [3], but few clinical data on SDT have been published. One reason is the difficulty in developing a sonosensitizing agent suitable for clinical use. Another reason is lack of an available systemic SDT device and a suitable clinical SPDT protocol. In recent years, a novel sonosensitizer was developed by the late Donald Burke, MD, of Advanced Technologies, Boston, USA. He named this Sonoflora $1^{\mathrm{TM}}$ (SF1). This agent is a chlorophyll

derivative with very high sonodynamic as well as photodynamic activity. Embryonic zebra fish assay has demonstrated that there is no evidence of toxicity associated with SF1 [5]. Our own animal studies have demonstrated that SDT with SF1 inhibits the growth of mouse S-180 sarcoma, even when the tumor is covered by a bone [6].

SDT is understood to produce cavitation around the surface of cancer cells, with the energy released following the collapse of the cavitating bubbles creating sonoluminescent light in the cancer cells [4]. The sonosensitizer is activated to an excited state from its ground state, and upon return to its ground state, released energy is transferred to the circumambient oxygen. This produces a large amount of reactive oxygen species (ROS) which mediate mitochondrial-dependent cell apoptosis by damaging the mitochondrial membrane and causing the release of Cytochrome $\mathrm{C}$ [4]. For a comprehensive explanation of the potential mechanisms by which SDT works see Wan et al. [4].

In vitro research has demonstrated that SPDT was able to generate more serious cytotoxicity in breast cancer cells than either SDT or PDT alone, and that ROS was significantly increased with the combined therapy [7]. In-vivo animal research in a highly metastatic 4T1 mouse xenograft model of breast cancer demonstrated that SPDT markedly inhibited tumor volume 
and tumor weight growth, suppressed tumor proliferation and significantly inhibited tumor lung metastasis, whilst not adversely impacting on body weight or major organs [7]. Another study in an animal tumor model found that the use of two sensitizers, titanium oxide, $0.2 \%-\mathrm{TiO}_{2}$ nanoparticles for SDT and $1 \mathrm{mM}$ 5 -aminolevulinic acid for PDT resulted in the best combination to achieve therapeutic effects [8].

Whilst many ultrasound devices are small, hand-held instruments, a whole-body ultrasound bath has been developed in order to reach all regions of the body. Potential advantages of this whole-body technology are that in addition to being able to address multiple cancer sites, it may also be able to impact on circulating tumor cells. In this pilot study in humans, we investigated the efficacy of a new systemic tumor therapeutic approach, SPDT incorporating whole body PDT and SDT with photo- and sono- sensitizers derived from chlorophyll, in combination with low-dose chemotherapy, as an adjunct or salvage treatment in advanced refractory breast cancers.

\section{Methods}

\section{Sensitizers}

The sensitizers used in this study were chlorophyll derivatives, SF1, SFa and UF. SF1 and SFa have average molecular weights of 942Da and 939Da respectively, but they have the same absorption spectrum with absorption peaks at wavelengths $402 \mathrm{~nm}$ and 636nm (Figure 1). UF has an average molecular weight of 660 and its absorption spectrum has three absorption peaks at wavelengths $414 \mathrm{~nm}, 629 \mathrm{~nm}$ and $677 \mathrm{~nm}$ (Figure 2).

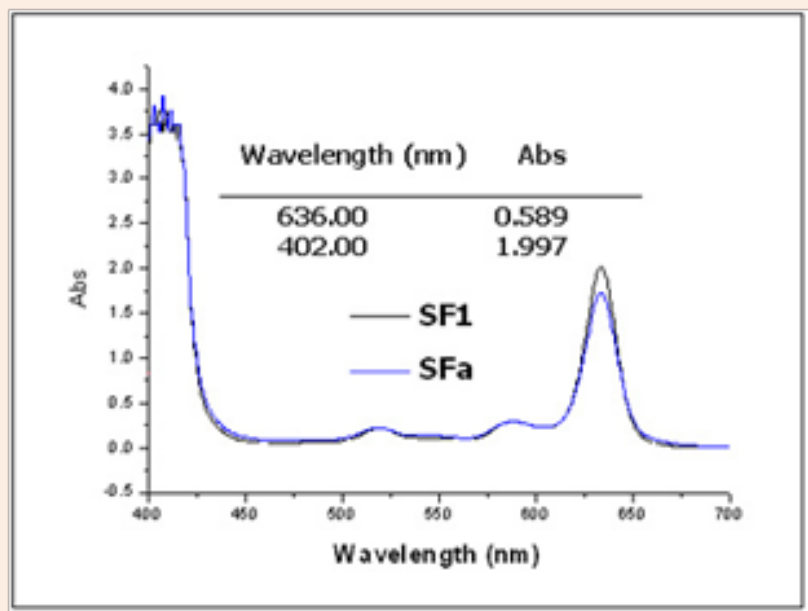

Figure 1: SF1 and SFa absorption.

\section{Systemic SDT equipment}

The new systemic SDT equipment is the Ultrasono-155I which has a total of 153 ultrasonic heads, with operating modes of continuous and pulse waves. The power output is $380 \mathrm{~V}$ and the frequency is $1.00 \mathrm{MHz} \pm 10 \%$. The device is like a bathtub, the total size of which is $2130 \mathrm{~mm} \times 1300 \mathrm{~mm} \times 1500 \mathrm{~mm}(\mathrm{LxWxH})$. See Figure 3. The bottom of bathtub is equipped with 125 ultrasonic heads formed in the shape of an adult human (Figure 3A). The bathtub is filled with sufficient water at $33-34^{\mathrm{L}}$ before the patient gets into it. The patient's position is determined according to the treatment requirement, that is, they are either floating in the water face up or face down. The upper ultrasonic device above the bathtub is equipped with 28 ultrasonic heads, as shown in Figure $3 \mathrm{Aa}$. It can be lowered down slowly and automatically ceases descending once it touches the water surface. It can also move horizontally and stop anywhere in the bathtub in order to cover the target zone (Figure 3B). Each of the 153 ultrasonic heads on the bottom and upper ultrasonic device are controlled by computer and emit non-focused ultrasound at the same frequency, pulse and intensity. Before clinical use, one of the study authors took part in a test seen in Figure 3Ca. He positioned himself in the water in the bathtub with the bottom ultrasound heads switched on; ultrasound transmitted through the body from the back and was detected by a medical ultrasonic diagnostic apparatus in the front part of the body including the chest. Conduction efficiency was found as follows: four limbs >crotch and shoulder >liver> abdomen and head >lungs. Figure $3 \mathrm{Cb}$ shows the ultrasound in the anterior chest transmitted from the back.

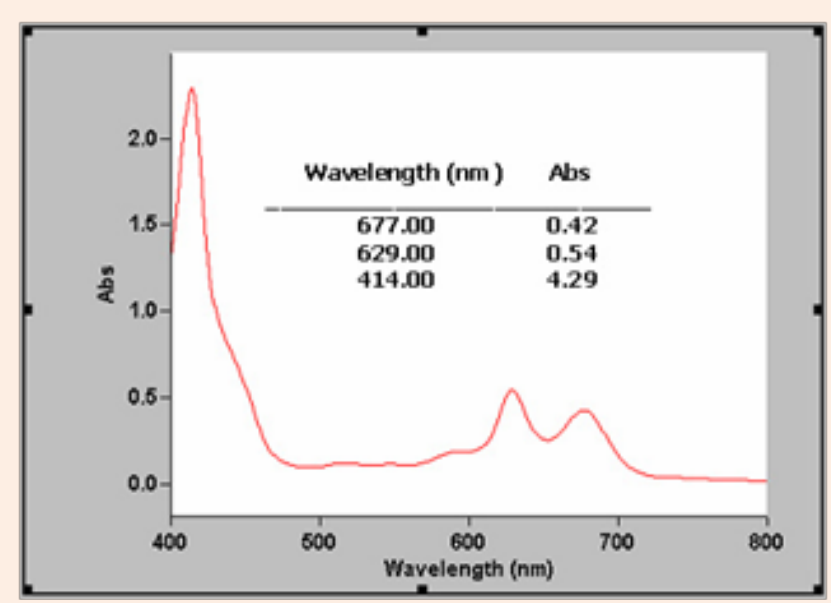

Figure 2: UF absorption scans: “Chem Lab” instrument.

\section{Systemic PDT equipment}

The PDT machine is manufactured by EEC Biotech Co. It uses LED red light at wavelength $554 \mathrm{~nm}$, with power of $220 \mathrm{~V}$. The size of the machine in which the patient lies, is $2400 \times 1300 \times 1400 \mathrm{~mm}$ $(\mathrm{LxWxH})$. The machine is almost fully enclosed. The patient lies supine on the 'bed' which has LED diodes underneath, and at the sides and above the patient.

\section{Study design and patients}

We reviewed the medical records of patients with advanced breast carcinoma patients who received SPDT or SPDT with dose-controlled chemotherapy at You Hao Hospital (located in Guangzhou, China) and Renkang Hospital (located in Guangdong, China) from May 2005 to November 2011. Eligibility criteria included: 1) pathologically-documented breast cancer; 2) presence of some existing tumor masses 3) no life-threatening complications such as serious jaundice and kidney failure, etc. The study was approved by the Renkang Hospital Human Research 
Ethics Committee, Guangdong. All patients were fully informed about the study and signed informed consent forms agreeing to participate in the study.

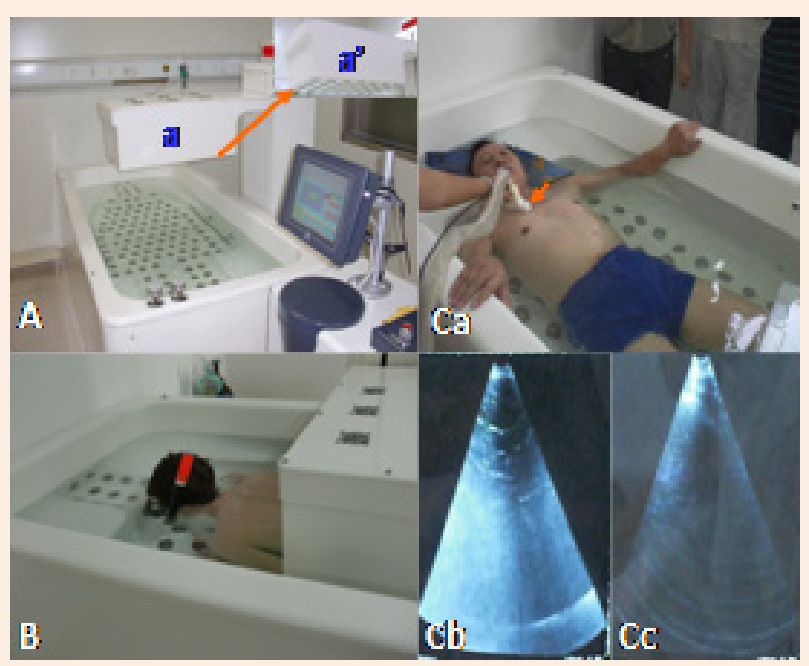

Figure 3: A new systemic SDT device.

A: The new systemic SDT device; Aa: upper ultrasonic device, Aa' is same as Aa, but showing 28 ultrasonic heads equipped on the bottom of the upper ultrasonic device. B: A patient being treated in the new systemic SDT device. Ca to Cc: A test of ultrasonic conduction efficiency through human body. Ca: The picture shows testing position and method, the arrow points to the diagnostic ultrasound probe. $\mathbf{C b}$ : The ultrasound picture when the bottom ultrasound heads are switched on. Cc: The ultrasound picture when the bottom ultrasound heads are switched off.

\section{SPDT Treatment}

The photosensitizer UF and either SFa or SF1 (both sonosensitizers) were given to patients sublingually on Day 1 and Day 2. Day 3 was a rest day. Days 4-6 inclusive were the SPDT treatment days: both PDT and SDT therapies were given, one session per day (of each). Day 7 was a rest day. The treatment was repeated the following week.

Two weeks of this 7- day protocol is considered one 'cycle' of SPDT Therapy. This was then followed by a rest week (no therapy). A 'round' of treatment typically included 1-4 cycles of SPDT.

For the PDT treatment, the tumor area and the whole body were irradiated by red LED light at $45 \mathrm{mV} / \mathrm{cm}^{2}$ and $554 \mathrm{~nm}$ of wavelength for 30 minutes. This was followed by SDT treatment. In the first three cases, a portable ultrasound device was applied to treat each tumor area for 20 minutes at $1 \mathrm{MHz}$ and $2.0 \mathrm{~W} / \mathrm{cm}^{2}$. In the other nine cases, using multiple ultrasound transducers within a systemic SDT device (SDT bath), the ultrasound was applied to the tumor area and whole body for 40 minutes at $75 \%$ pulse, $1 \mathrm{MHz}$ and $2.0 \mathrm{~W} / \mathrm{cm}^{2}$.

Nine patients had concurrent chemotherapy with dosages ranging from moderate to low (in the order of $1 / 4$ conventional dosages), the dosage selected to keep side effects at Grade II or better.
To reduce the side effects and increase the efficacy of SPDT, patients underwent Ozone Therapy before SPDT and were given oxygen supplementation (middle flow supplementary oxygen) during SPDT.

\section{Efficacy outcome variables}

The primary outcome variable was Response Rate (RR). Subcategories of this were: Complete Response (CR), Partial Response (PR), and Stable Disease (SD) according to the Revised Response Evaluation Criteria in Solid Tumors (RECIST) Criteria (version 1.1) [9]. The criteria are as follows:

a. Complete Response (CR): Disappearance of all target lesions. Any pathological lymph nodes (whether target or non-target) must have reduction in short axis to $<10 \mathrm{~mm}$.

b. Partial Response (PR): At least a $30 \%$ decrease in the sum of diameters of target lesions, taking as reference the baseline sum diameters.

c. Progressive Disease (PD): At least a $20 \%$ increase in the sum of diameters of target lesions, taking as reference the smallest sum on study (this includes the baseline sum if that is the smallest on study). In addition to the relative increase of $20 \%$, the sum must also demonstrate an absolute increase of at least $5 \mathrm{~mm}$. (Note: the appearance of one or more new lesions is also considered progression).

d. Stable Disease (SD): Neither sufficient shrinkage to qualify for PR nor sufficient increase to qualify for PD, taking as reference the smallest sum diameters while on study'[9].

The secondary outcome variables were overall survival (OS) and side effects (toxicities) (see next section on safety and tolerability). Overall survival was calculated from the first day of SPDT.

Patient assessment included a complete medical history, physical examination, and laboratory studies every SPDT cycle and imaging studies every two SPDT cycles in accordance with the RECIST criteria [9]. PET/CT scans or CT scans were performed on all patients before SPDT and after last the SPDT cycle to precisely evaluate the results.

\section{Safety and Tolerability}

Safety was assessed using Full Blood Examination, liver function, and renal function tests. Toxicity was graded using the National Cancer Institute's (NCI) Common Terminology Criteria for Adverse Events v3.0 (CTCAE v3.0) [10].

\section{Statistics}

Efficacy results are reported simply as descriptive statisticsproportion of patients who achieved either Complete Response, Partial Response, Stable Disease or Progressive Disease status, in accordance with the definitions given in the previous section. Safety and tolerability is reported as a proportion of total participants assessed as having Grade 1, 2, 3 or 4 toxicity in relation to tumor-site pain, fatigue, neutrophil count and platelet count in accordance with the NCI Common Terminology Criteria for Adverse Events v3.0. 
Results

\section{Efficacy and Overall survival}

Twelve patients with advanced breast carcinoma participated in the study (11 females and 1 male), ten with metastases in the viscera, one in the brain, and nine within bone. Ten had undergone prior chemotherapy and nine had failed at least second lines of conventional chemotherapy.

At a mean follow-up period of 34 months (range 9-68 months), after the first round of treatment the twelve patients had received an average of 3.25 cycles (range 1-4 cycles) of either SPDT plus low-dose chemotherapy $(n=9)$ or SPDT therapy alone $(n=3)$. The overall response rate after the first treatment round was 75\%, with two patients achieving Complete Response (16.7\%) and seven patients achieving Partial Response (58.3\%). The remaining three patients (25.0\%) achieved a Stable Disease state.

In those three patients who used SPDT as sole therapy, two achieved positive results (Partial Response) after one treatment round with the other achieving Stable Disease state. One patient who had metastases to both viscera and brain was responsive to the treatment, achieving a Partial Response, shown in Figure 4.

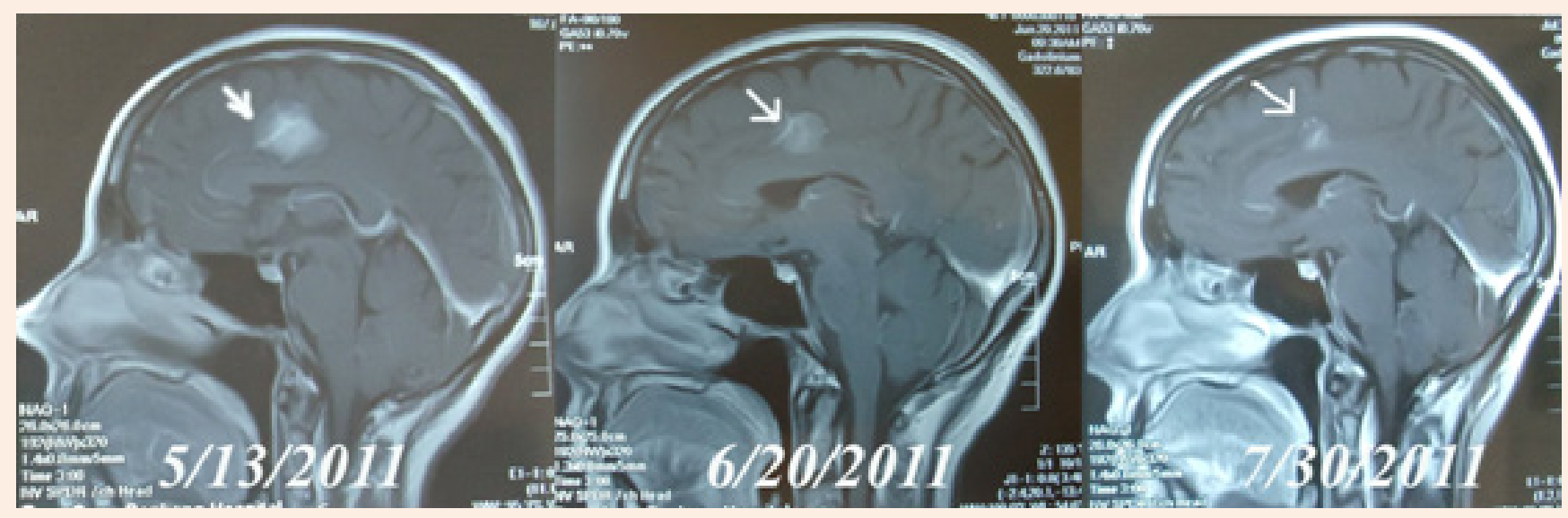

Figure 4: MRI brain scans in Case 10. A female, 46 years old had advanced breast cancer with brain metastasis. Figure 5/13/2011 is MRI before treatment.

Figure 6/20/2011 is the MRI after the second cycle of SPDT treatment: the arrows show the same brain lesion. After 4 cycles of SPDT/dose controlled chemo (Initial Dose: Liposomal Doxorubicin 30mg/M² + Paclitaxel Liposome 120mg/M²), the MRI on 7/30/2011 showed PR of the brain mass.

Five of the 12 patients required second or third rounds of treatment with SPDT after their tumors relapsed, and these patients achieved positive results: four achieved Partial Response and one Stable Disease. However, eventually all 12 patients died. The mean overall survival time for the twelve women was $25+$ /18 months (range 6 - 68 months).

\section{Safety and Tolerability}

In general, SPDT plus dose-controlled chemotherapy was found to be well tolerated. SPDT plus dose-controlled chemotherapy produced some side effects including fatigue and evidence of bone marrow suppression. Generally speaking, cancer patients with KPS $\leq 50$ are not suitable for conventional chemotherapy. In this study, four patients with KPS $\leq 50$ had SPDT plus dosecontrolled chemotherapy. They all tolerated this well; even Case 1 , a terminally ill patient with KPS 10 was treated safely and effectively [11]. Treatment-related side effects and toxicity grade in accordance with the NCI Common Terminology Criteria for Adverse Events v3.0. for all patients combined is set out in Table 1.

Table 1: Treatment-Related Side Effects and Toxicity of All Patients.

\begin{tabular}{|c|c|c|c|c|c|c|}
\hline \multirow{2}{*}{ Adverse Event } & \multicolumn{5}{|c|}{ Toxicity Grade* } & \multirow{2}{*}{ Total \% } \\
\cline { 2 - 6 } & $\mathbf{1}$ & $\mathbf{2}$ & $\mathbf{3}$ & $\mathbf{4}$ & $\mathbf{5}$ & \\
\hline Tumor site pain & 0 & 2 & 0 & 0 & 0 & $16.7 \%$ \\
\hline Fatigue & 5 & 2 & 1 & 0 & 0 & $66.7 \%$ \\
\hline Neutrophil count & 1 & 2 & 1 & 0 & 0 & $33.3 \%$ \\
\hline Platelet count & 1 & 1 & 0 & 0 & 0 & $16.7 \%$ \\
\hline
\end{tabular}

*NCI Common Terminology Criteria for Adverse Events v3.0. 


\section{Discussion}

This is the first exploratory study of the efficacy of SPDT using whole body or systemic SDT plus whole body PDT. This novel SDT technology utilises a bath with 125 ultrasonic heads, wherein the patient lies in water in order to allow the ultrasound to penetrate the whole body. In this way, multiple tumors may be treated simultaneously. It is also postulated that it may also have an impact on circulating tumor cells. Further research would be needed to ascertain this.

The majority of participants were treated with low-dose chemotherapy in conjunction with SPDT. Low dose chemotherapy has the advantage of providing less of a toxic insult to the body compared to conventional doses. The major treatment-related side effects were fatigue in $75 \%$ of participants, and in the majority of cases, this was Grade 1 toxicity. In two cases, there was a change in platelet count and in four cases, a change in neutrophil count. Overall, results suggest that the SPDT plus chemotherapy was well tolerated and not associated with significant toxicity, as assessed using the NCI Common Terminology Criteria for Adverse Events v3.0.

The limitations of this study are that it was a single-arm study with no control. In addition, numbers of participants are low. Results have been reported as simple proportions only, in accordance with the definitions for efficacy defined previously. Nonetheless, as an exploratory pilot study, it does provide an indication that SPDT plus low dose chemotherapy, or where patients can't tolerate or choose not to have chemotherapy, SPDT alone, may be a useful therapy to reduce tumor masses and at least prolong survival. Studies in a larger population of patients in the future will be necessary.

The mean overall survival time was $25+/-18$ months, with one patient surviving 68 months. All eventually died. However, on entering this study, these women had advanced refractory breast cancer at the time they entered the study. The earlier that cancer can be treated, the better. What is important in terms of achieving longer term outcomes in patients is addressing the factors that may have led to the cancer in the first place, and supporting the patient during and after treatment with a variety of therapies [12]. A healthy patient with cancer will generally do better than an unhealthy one. Diet, sleep, vitamin D, exercise and stress reduction are all important to address in the holistic management of the cancer patient [12]. Chinese herbal medicine, western herbal medicine and other forms of complementary medicines have been shown to assist in the management of cancer [12]. Further studies are needed to assess the efficacy of SPDT with or without low-dose chemotherapy with the addition of a holistic, treatment care plan that includes addressing the factor that can contribute to cancer and that includes supportive complementary therapies [12]. Further studies in women with early breast cancer and studies in other forms of cancer are also needed.

\section{Conclusion}

This study indicates that SPDT with low-dose chemotherapy can be used more safely, effectively and repeatedly in patients with advanced refractory breast cancer. SPDT with and without lowdose chemotherapy shows potential as a new systemic approach for tumor adjunct therapy, which merits further investigation.

\section{Acknowledgment}

None.

\section{Conflict of Interest}

The authors declare no conflict of interest.

\section{References}

1. Dolmans DE, Fukumura D, Jain RK (2003) Photodynamic therapy for cancer. Nat Rev Cancer 3(5): 380-387.

2. Brancaleon L, Moseley H (2002) Laser and non-laser light sources for photodynamic therapy. Lasers Med Sci 17(3): 173-186.

3. Kinoshita M, Hynynen K (2006) Mechanism of porphyrin-induced sonodynamic effect: possible role of hyperthermia. Radiat Res 165(3): 299-306.

4. Wan GY, Liu Y, Chen BW, Liu YY, Wang YS, et al (2016) Recent advances of sonodynamic therapy in cancer treatment. Cancer Biol Med 13(3): 325-338.

5. Lewis TJ (2010) Toxicity and cytopathogenic properties toward human melanoma cells of activated cancer therapeutics in Zebra fish. Integr Cancer Ther 9(1): 84-92.

6. Wang X, Lewis TJ, Mitchell D (2008) The tumoricidal effect of sonodynamic therapy on S-180 sarcoma in mice. Integr Cancer Ther 7(2): 96-102.

7. Liu Y, Wang P, Liu Q, Wang X (2016) Sinoporphyrin sodium triggered sono-photodynamic effects on breast cancer both in vitro and in vivo. Ultrasonics Sonochemistry 31: 437-448.

8. Miyoshi N, Kundu SK, Tuziuti T, Yasui K, Shimada I, et al. (2016) Combination of sonodynamic and photodynamic therapy against cancer would be effective through using a regulated size of nanoparticles. Nanosci Nanoeng 4(1): 1-11.

9. Eisenhauer EA, Therasse P, Bogaerts J, Schwartz LH, Sargent D, et al. (2009) New response evaluation criteria in solid tumors: revised RECIST guideline (version 1.1). Eur J Cancer 45(2): 228247.

10. https://ctep.cancer.gov/protocolDevelopment/electronic applications/docs/ctcaev3.pdf

11. Wang X, Zhang W, Xu Z, Luo Y, Mitchell D, et al. (2009) Sonodynamic and Photodynamic Therapy in advanced breast carcinoma: A report of three cases. Integr Cancer Ther 8(3): 283-287.

12. O’Brien KA, Sali A (2017) A Clinician's Guide to Integrative Oncology: What You Should be Talking About with Cancer Patients and Why. Springer, USA. 